\title{
A Matter of Trust? Examination of Chatbot Usage in Insurance Business
}

\author{
Davinia Rodríguez Cardona \\ Leibniz Universität Hannover \\ rodriguez@iwi.uni-hannover.de
}

Michael H. Breitner

Leibniz Universität Hannover

breitner@iwi.uni-hannover.de

\author{
Antje Janssen \\ Leibniz Universität Hannover \\ janssen@iwi.uni-hannover.de
}

\author{
Nadine Guhr \\ Technische Hochschule \\ Ostwestfalen-Lippe \\ nadine.guhr@th-owl.de \\ Julian Milde \\ Leibniz Universität Hannover \\ $\underline{\text { milde@iwi.uni-hannover.de }}$
}

\begin{abstract}
Critical success factors such as trust and privacy concerns have been recognized as grand challenges for research of intelligent interactive technologies. Not only their ethical, legal, and social implications, but also their role in the intention to use these technologies within high risk and uncertainty contexts must be investigated. Nonetheless, there is a lack of empirical evidence about the factors influencing user's intention to use insurance chatbots (ICB). To close this gap, we analyze (i) the effect of trust and privacy concerns on the intention to use ICB and (ii) the importance of these factors in comparison with the widely studied technology acceptance variables of perceived usefulness and perceived ease of use. Based on the results of our online survey with 215 respondents and partial least squares structural equation modelling (PLS-SEM), our findings indicate that although trust is important, other factors, such as the perceived usefulness, are most critical for ICB usage.
\end{abstract}

\section{Introduction}

The rapid development of artificial intelligence (AI) and natural language processing (NLP) has contributed to the expansion of the use of conversational agents in recent years [1]. In almost all areas of life, such as education, entertainment and health, chatbots are increasingly being used [2] and, already over 75 million inquiries were addressed worldwide by chatbots in 2019 alone [3]. To remain competitive, companies in traditional sectors are transforming their customer experience by building digital strategies around new digital services, products and interaction channels enabled by the introduction of technological innovations [4].
In the insurance sector, however, it is much more difficult to adopt new technologies such as chatbots than in other application areas due to various legacy regulations for online systems $[5,6]$. This is because, in data-rich contexts with high levels of sensitive data, as in the insurance sector, the safe processing of customer-related data and the protection of privacy are major challenges to prevent that customers may suffer harm through financial loss or physical and psychological damage [7]. As a consequence, the relevance of trust and privacy concerns is emphasized in insurance-related tasks. However, while strict legacy regulations mainly influence the development and implementation of ICB, it is the underlying mechanism building the perceptions and preferences of the users which decide whether or not a human-robot interaction through chatbots will be accepted in the insurance business. Nonetheless, there is limited empirical evidence about the subjective factors underlying the potential and experienced users' behavioral intentions to interact with ICB. Hence, to contribute to a better understanding of these factors, using internet users in Germany with previous chatbot experience as the unit of analysis, we examine (i) the effect of privacy concerns and trust in chatbot systems, and (ii) the importance of these factors in comparison with the widely used Technology Acceptance Model (TAM) variables of perceived usefulness (PU), and perceived ease of use (PEOU). Based on our results of a survey with 215 respondents and PLS-SEM, we address the following research question (RQ):

How do trust, privacy concerns, perceived ease of use, and perceived usefulness effect the intention to interact with ICB?

After presenting related literature to ICB, trust, privacy concerns and their interplay with technology acceptance, we derive our hypotheses from academic literature and develop a conceptual model that 
combines technology acceptance with privacy and trust-related factors, followed by a description of our research design and methodology of the survey. After that, we outline the data analysis results followed by our discussion, limitations and implications for research and practice.

\section{Theoretical background and hypotheses}

\subsection{Chatbots in the insurance business}

Chatbots, also known in the scientific literature under the term "conversational agents" $[2,8]$ are interactive intelligent systems that use NLP and machine learning techniques (ML) to conduct textbased conversations with humans about a particular topic over a natural language-based user interface [6]. The ability to automate and simplify existing complex processes is one reason why chatbots are increasingly being developed by companies in the insurance sector $[6,9,10]$. Generally, in the insurance sector, customer contact usually exists for two reasons, namely the conclusion of an insurance contract or the notification of a claim [11]. Both aforementioned areas could benefit from several advantages using chatbots such as $24 / 7$ availability and fast processing. Given the fact that many people are against the paperwork associated with an insurance contract, chatbots could help to simplify this process, for example by enabling the chatbot to use simple language to explain complicated contractual terms in insurance policies [10]. Singh et al. [10] stated that more than $70 \%$ of the requests in insurance companies are characterized by basic queries such as current claim status, account information or policy information which can also be automated via a chatbot.

At the same time, insurance companies are driven by customer data, so that risk profiles and the appropriate insurance options can be determined precisely. Given that insurance companies are becoming increasingly similar, customer data is seen as a key factor in adapting services to individual needs [12]. By communicating and exchanging information with the customer, chatbots offer new cost-effective ways to influence the customer's value creation process within the dialog and present individualized offers $[11,12]$.

To assess the practical status quo of chatbot design elements and application areas in the German insurance sector, we identified six chatbots in German language (Claim Assistant by VGH, Lizzy by HDI, Travel Assistant by ARAG, ServiceBot by Vienna Insurance Group, Digital Assistant by Admiral Direkt and Clara by Helvetia) across 40 monitored insurers available in Germany (See Online-Appendix Table A1,
http://bit.ly/Online_Appendix). To get a detailed understanding of $\overline{\mathrm{ICB}}$ design features, we classified them according to the chatbot taxonomy of Janssen et al. [2]. The analyses have shown that these ICB are all goal-oriented, rule-based customer service chatbots, which mostly do not show any socio-emotional behavior. Within the conversation, four ICB help the user in the role of an expert and two as a facilitator. Three ICB are represented by an avatar. Four ICB have a graphical interface with selection buttons and two ICB offer the user to directly type a sentence [2]. The chatbots are offered for use on the websites of the respective insurance companies to guide through claim processes of e.g., bicycle thefts or to calculate suitable insurances for personal liability or households, among others (for more information see Online-Appendix Table A2). All of them have the possibility to get additional human support. We further discovered that only two out of six chatbots referred to the privacy policy and asked the user for confirmation at the beginning of the conversation. Contrary, it is remarkable that three chatbots asked for personal data such as car registration number, name, birth date or insurance number. Another chatbot asked very sensitive questions about insurance coverage preferences, such as whether one would like to get alternative treatment methods paid within the insurance contract (Table A2). This querying of very sensitive data distinguishes ICB from chatbots in other application areas, e.g., daily life or entertainment [2]. To reveal this sensitive personal data within a conversation, users must have a certain amount of trust to the ICB which depends not only on the reputation of the company, but above all on the acceptance of the technology. Rather, a customer's decision to disclose their data depends on their individual privacy experiences, the benefits anticipated, and how the data is processed [13]. Therefore, there is a need for an empirical investigation of ICB acceptance in relation to trust and privacy concerns.

\subsection{The notion of trust and its dimensions}

From a traditional social psychology perspective, trust has been defined by Rousseau et al. [14, p. 395] as "a psychological state comprising the intention to accept vulnerability based upon positive expectations of the intentions or behavior of another". This conceptualization of trust is largely related to the existence of a theoretical conditional-based willingness to depend on another party, in order to offset the existence of uncertainty and risk in the context of human-to-human relationships [14].

As indicated by Fan et al. [15], in the scientific literature, the nature of trust is envisioned either as a 
one-dimensional or as a multi-dimensional social construct. The unidimensional conception of trust (known as cognitive or character-based trust), embodies a logical trust construct which is based on a rational judgment upon the characteristics or qualities that the entrusted party possesses, such as integrity, trustworthiness, benevolence or specific abilities, and whose potential benefits are expected to outweigh the risks associated with the interaction [15]. While the multi-dimensional representation of trust (defined as affective or relationship-based trust) comprises not only cognitive, but also affective dimensions formed through emotional affection or mutual identification [15]. For this analysis, we take a onedimensional cognitive perspective of trust, in which the aggregate effect of the rational judgments upon the characteristics of an ICB comprises the trust construct.

\subsection{Trust and privacy concerns in chatbot systems}

Some studies such as Jian [16] have determined that there are no significant differences in the components of trust in a comparison of trust between human to human, human to machine, and trust in general. However, McKnight et al. [17] argued for a distinction between trust in technology and trust in people and further addressed the need to isolate and identify factors affecting trust in technology. In that regard, Large et al. [18, p. 49] consider trust in technology, embodied in "the extent to which people believe that technology will perform effectively and without a negative or injurious outcome", to be critical for the information technology (IT) acceptance at the user level. On that account, recent studies have focused on examining different aspects related to trust or privacy concerns with regard to chatbots (e.g. [19-22]). For instance, through an exploratory interview study, Følstad et al. [19] examined the determinant trust factors for customer service chatbots from a user perspective. Based on their interview results, they identified the ability of the chatbot to correctly understand the user and provide effective advice as the key trust factors for customer service chatbots. While Laumer et al. [20] found out that a higher level of trust in the provider and the technology in healthcare chatbots can minimize the subjectively perceived privacy risk of a user. Furthermore, Kasilingam [21] analyzed the intention to use e-commerce chatbots by combining the TAM and diffusion of innovations theory. The study showed that only trust had a direct influence on the intention to use shopping chatbots. Likewise, Rese et al. [22] investigated the factors influencing the intention to use shopping chatbots using TAM and the uses and gratification theory. The results shown that privacy concerns have a significant negative impact on the intention to use shopping chatbots even when these do not sell products directly.

Insurance chatbots differ from the previously examined chatbot application areas, e.g. e-commerce, in that they (i) handle claim processes as well as market complex, and individualized insurance products which require explanation and (ii) the disclosure of sensitive information, such as income, work or medical history and health habits. This demands a consideration of technology acceptance factors such as ease of use as well as trust and privacy concerns. However, the combination of trust and privacy concern factors and their intersection with TAM constructs has received limited attention in relation to chatbots [21, 22]. Despite the fact that the successful use of chatbots can offer unique opportunities for insurance companies $[2,10]$, there is no study that examines trust and privacy concerns in relation to the subjective acceptance factors underlying the users' behavioral intentions to interact with ICB and offer design implications for their future development.

\subsection{The interplay between chatbot acceptance, trust and privacy concerns}

The most used theoretical model for identifying and predicting the factors that drive the intention to use and the acceptance of technological innovations is the TAM of Davis [23], which has been the base for theoretical extensions such as the TAM2 [24] and the unified theory of acceptance and use of technology (UTAUT) model [25]. Given that TAM offers a broad spectrum of questions related to acceptance factors [21] and allows a robust application into several contexts while remaining on a general level (ease of use and perceived usefulness) [22, 24], we decided to apply TAM within this analysis. In previous studies, TAM was used in its original version as well as in combination with other variables. But an extension of the model to include technology-specific external variables $[21,23,26]$ is recommended to strengthen the predictive and explanatory power [27]. We have done this by including the variables trust and privacy concerns, following the approach of previous studies on trust and technology acceptance (see e.g., $[5,21,26]$ as well as on privacy concerns and technology acceptance (see e.g., [22, 26, 28] in diverse application areas.

According to Fishbein and Ajzen [29], it is essential that the newly included variables are consistent with the existing ones, when extending an established model [26]. Along the lines of the Theory of Reasoned Action (TRA) and the Theory of Planned Behavior (TPB), the perceived usefulness and the 
perceived ease of use of a technology are the two critical internal beliefs for the user acceptance. These two beliefs in turn can be influenced by external or moderating factors, such as user characteristics or system features [29]. The variables privacy concerns and trust are also seen on the same level of abstraction as ease of use and perceived usefulness [26], since they also measure general beliefs about a technology, i.e., ICB, without specifying deeper details about individual features of a chatbot.

In the context of our research, we define the intention to interact with ICB as the desire of a potential or current insurance customer to use a computerized text-based dialog system to accomplish a specific insurance-related goal or task. Empirically, the relevance of perceived ease of use has been proven by various studies (see e.g., [5]). Likewise, some recent studies, such as Müller et al. [8] have underlined the role of the discernible level of naturalness in the interaction with chatbots as a mediating variable for their acceptance. This leads us to conjecture that if the operation of an ICB is simple, clear, understandable and easy to learn, then the operational characteristics of the chatbot have the potential to positively impact the internal believes of the users and consequently, to affect the behavioral intentions of the users in favor of the use of chatbots in the insurance business [30]. Accordingly, we have formulated the following hypothesis:

$H_{1}$ : The perceived ease of use is positively related to the intention to use ICB.

On the other hand, other operational variables such as the problem-solving competences of chatbots can condition the disposition of the users to interact with them [9]. Recent research on the analysis of the intention to use financial technology innovations such as blockchain, biometric authentication, robo-advisors and peer-to-peer platforms, determined that a higher synchronism readiness, that is, the capacity of the technology to enable higher process efficiency for the users through seamless, fast or instant transactions independent from human employee processing [30], positively mediates the intention to use new technology and outweighs potential constricts inherent to humancomputer interaction (HCI) such as a lower social presence [30]. Therefore, a chatbot is used more extensively if it is subjectively positive rated by the user because its operational characteristics adequately support the user to accomplish an insurance-related goal or task, by offering a faster and efficient advice or detailed insurance knowledge without constrains of time or location [31]. Based on this assumption, we derive the next hypothesis:

$\mathrm{H}_{2}$ : The perceived usefulness is positively related to the intention to use ICB.
An influence of perceived ease of use on perceived usefulness has been theorized by Davis [23]. When using a chatbot, users expect from them to properly rationalize textual input and to have sufficient domainspecific linguistic knowledge to provide suitable responses or actions [1]. Wuenderlich and Paluch [9] discuss the importance of highlighting the service competence and outcome of a chatbot at an early stage of the interaction, as this factor has an impact on the authenticity perception and problem-solving competence of the chatbot as experienced by users during communication exchange. To empirically assess this supposition, we derive the following hypothesis:

$\mathrm{H}_{3}$ : The perceived ease of use is positively related to the perceived usefulness of ICB.

In addition, McKnight et al. [17] recommend investigating operational components, such as the constructs belonging to the TAM, in combination with trusting beliefs, since trusting beliefs have an impact on the intention to engage in trust-related activities. Therefore, the users with positive trusting views assume that the technology has positive and desirable attributes [17]. User trust is seen by several researchers as a key factor for the adoption of a new technology in contexts such as e-commerce [26] and e-services [32]. In view of the above, we deduce that trust creates positive attitudes and perceived behavioral control towards interaction with ICB, offsets uncertainty and creates expectations of a positive outcome, which in turn, has a positive effect on the intention of chatbot users to use chatbots. Consequently, we derive the following hypothesis:

$\mathrm{H}_{4}$ : Trust is positively related to the intention to use ICB.

Furthermore, an influence of trust on perceived usefulness was observed by Gefen and Straub [32]. Lankton et al. [33] tested the influence of system-like trust on the perceived usefulness, i.e. perceived value of technology usage. By taking Facebook and Microsoft Access as sample technologies, the researchers were able to detect a significant influence of system-like trusting beliefs on perceived usefulness in both technologies, even although this influence was of varying intensity. We now refer this outcome to the chatbot context, which results in the following hypothesis:

$H_{5}$ : Trust is positively related to the perceived usefulness of ICB.

The influence of perceived ease of use on trust is described in the literature as a basis for users to assess whether and to what extent they can trust another party [32]. For instance, Gefen and Straub [32] identified a link between trust and perceived ease of use and attributed it to the fact that the online merchants investigated in the study, invested in the 
relationship with customers by ensuring good ease of use. Hence, since the insurer also invests in the customer relationship by giving users a user-friendly experience and a sense of control over the chatbot interaction process, we conjecture that:

$H_{6}$ : The perceived ease of use is positively related to trust in ICB.

Privacy in the context of HCI research has been considered as one of the grand challenges for the research field of intelligent interactive technologies [34]. Stephanidis et al. [34, p. 1232] defined privacy as "the ability of users to be in control and to determine what data can be collected and exploited by a computer system and then be shared with third parties". Past research has demonstrated that the psychological, emotional, and relational effects of disclosing personal information to a chatbot are the same that providing information to a human [35]. As chatbot users may not be willing to disclose personal information if they are unsure about security aspects or suspicious of the chatbot [7], we derive the following hypothesis: in ICB.

$H_{7}$ : Privacy concerns are negatively related to trust

Diverse privacy concerns have been found to negatively influence the intention to use new technologies across diverse application areas, e.g. smart home [27] and smartwatch [28]. Considering privacy concerns as the perceived risk that the user's personal or financial information could be processed, collected and used by third parties in a manner that is unauthorized, harmful or contrary to the interests of the user, we expect similar effects on the intention to use ICB due to the sensitivity of insurance data which is why we derive the last hypothesis:

$H_{8}$ : Privacy concerns are negatively related to the intention to use ICB.

Although the focus of our study is on understanding the subjective factors underlying the users' behavioral intentions to interact with ICB, it can be suggested that the experience with ICB may play a role in judging how much a participant may trust an ICB. To provide a robust model, we therefore considered experience and used it as covariate. We incorporate experience as covariate or counterargument, but we take no definite stand regarding their predicted direction.

\section{Methodology and research design}

To collect empirical data to operationalize the previously conceptualized constructs, we performed a standardized online cross-sectional survey. The survey questionnaire consisted of closed questions related to 22 measurement items. In agreement with the objective of the study, the measurement items were arranged into the categories of trust, privacy concerns, perceived ease of use, perceived usefulness and, intention to use. In addition to the measurement items, some demographic (i.e., age and gender) and chatbot experience queries were incorporated into the survey instrument. The survey instrument and the corresponding measurement scales are available in Online-Appendix Table A3.

Given that the scope of this analysis is focused on the specific context of Germany, the survey questionnaire was developed in German language to avoid translation bias or assessment inconsistencies due to communication barriers. The survey was made available through the online survey portal of Circle3, electronic mail, social media, and student platforms. The questionnaire included an introductory text to present the aim of the survey. The target group invited to participate were internet users living in Germany with previous chatbot experience in other domains. Through the aforementioned collection nodes, a total of 215 respondents completed the survey, of which $51.1 \%(n=110)$ were male and $48.9 \%(n=105)$ were female. The average age of the respondents corresponds to 29.97 years. Despite of their familiarity with chatbots in other domains, only about $9 \%$ of the respondents have previously used an ICB.

All measurement items were derived and adapted from prior scientific research (See Online-Appendix Table A3). For the assessment of trust in chatbot systems perceived by the participants, different scales of trust related to trusting beliefs were consolidated (e.g., $[7,17,33])$ and operationalized through semantic differential word pairs (i.e. trust and distrust factors) using a rating scale graded by five numerical values $[+2 ;+1 ; 0 ;-1 ;-2]$. The construct of privacy concerns was abstracted in terms of privacy and protection beliefs corresponding to risks of identity or data theft, unauthorized secondary used, and information control $[36,37]$. Furthermore, the conceptualization of the constructs of perceived ease of use, perceived usefulness, and intention to use were drawn from the literature on technology acceptance. The privacy concerns, as well as the acceptance constructs, were measured using a five-point Likert scale.

\section{Data analysis and results}

To test our theoretical assumptions against the collected empirical data and to identify the relationships underlying the intention to use chatbots in the insurance business, we used partial least squares structural equation modelling (PLS-SEM) [38]. PLSSEM is, in contrast to its sibling the covariance-based structure equation model, designed for complex scenarios with small sample sizes in which a number of 
different potential influences need to be tested using several indicators and multiple items [39].

For the computation of the structural model and the validation of the measurement model, we applied a two-step approach using the software SmartPLS version 3 [40]. At first, in line with Hair et al. [40], we assessed the item reliability and composite reliability of the construct measures, subsequently, we evaluated their convergent and discriminant validity. To assure the item reliability, we evaluated the outer loadings of each indicator in relation to their respective underlying latent variable by means of a bootstrapping procedure based on 3000 replications. The factor loadings should be equal to or greater than 0.707 , in order that at least $50 \%$ of the variance of each indicator is explained by their respective latent variable [38]. In our analysis, all outer loadings of the latent variables were above the aforementioned threshold value (Table 1).

Table 1. Outer loadings and cross-loadings

\begin{tabular}{|c|c|c|c|c|c|c|}
\hline Items & INT_USE & PEOU & PU & PRIV & TR & EXP \\
\hline INT_USE1 & $0.740 * * *$ & 0.225 & 0.499 & -0.249 & 0.371 & -0.076 \\
\hline INT_USE2 & $0.882 * * *$ & 0.317 & 0.636 & -0.172 & 0.417 & -0.086 \\
\hline INT_USE3 & $0.786 * * *$ & 0.228 & 0.444 & -0.273 & 0.394 & -0.066 \\
\hline PEOU1 & 0.357 & $0.891 * * *$ & 0.405 & -0.148 & 0.376 & 0.028 \\
\hline PEOU2 & 0.268 & $0.893 * * *$ & 0.352 & -0.087 & 0.334 & -0.045 \\
\hline PEOU3 & 0.244 & $0.925 * * *$ & 0.379 & -0.145 & 0.371 & -0.028 \\
\hline PEOU4 & 0.288 & $0.899 * * *$ & 0.351 & -0.191 & 0.358 & -0.001 \\
\hline PU1 & 0.557 & 0.342 & $0.789 * * *$ & -0.182 & 0.345 & -0.056 \\
\hline PU2 & 0.430 & 0.298 & $0.780 * * *$ & -0.164 & 0.345 & -0.026 \\
\hline PU3 & 0.503 & 0.301 & $0.814^{* * *}$ & -0.272 & 0.425 & -0.019 \\
\hline PU4 & 0.544 & 0.326 & $0.839 * * *$ & -0.278 & 0.441 & -0.024 \\
\hline PU5 & 0.563 & 0.306 & $0.756^{* * *}$ & -0.187 & 0.348 & -0.034 \\
\hline PU6 & 0.583 & 0.410 & $0.843^{* * *}$ & -0.311 & 0.411 & -0.051 \\
\hline PRIV1 & -0.245 & -0.130 & -0.249 & $0.883 * *$ & -0.422 & 0.006 \\
\hline PRIV2 & -0.267 & -0.150 & -0.261 & $0.873^{* * *}$ & -0.423 & 0.056 \\
\hline PRIV3 & -0.196 & -0.146 & -0.239 & $0.907 * *$ & -0.347 & 0.027 \\
\hline PRIV4 & -0.279 & -0.144 & -0.292 & $0.914 * *$ & -0.420 & 0.017 \\
\hline TR1 & 0.356 & 0.312 & 0.316 & -0.236 & $0.765^{* * *}$ & -0.060 \\
\hline TR2 & 0.387 & 0.244 & 0.377 & -0.424 & $\mathbf{0 . 8 2 0} * * *$ & -0.055 \\
\hline TR3 & 0.374 & 0.349 & 0.394 & -0.365 & $0.815 * * *$ & -0.054 \\
\hline TR4 & 0.403 & 0.356 & 0.408 & -0.368 & $0.723 * * *$ & -0.023 \\
\hline TR5 & 0.424 & 0.333 & 0.413 & -0.399 & $0.867 * * *$ & 0.054 \\
\hline EXP3 & -0.095 & -0.011 & -0.044 & 0.030 & -0.038 & $1.000 * * *$ \\
\hline
\end{tabular}

Afterwards, we evaluated the composite reliability (a.k.a. internal consistency reliability, ICR). The composite reliability is an indicator whose value indicates the extent to which the items of a construct measure diverse aspects of the same latent construct [41]. According to Diamantopoulos et al. [42] the ICR should be above a threshold value of 0.70 to assure composite reliability. Given that the composite reliability values of our measurement model range between 0.846 and 1.000 (Table 2), we concluded that the model possesses a high level of internal consistency reliability.

Consecutively, to evaluate the convergent validity, we calculated the average variance extracted (AVE). The AVE verifies how the construct reliably is represented by all items assigned to it [43]. As shown in Table 2, all latent variables reached the required satisfactory threshold of 0.5 [43], indicating that the measures of the latent variables show high levels of convergent validity. The capacity of the model to explain the variance of the indicators can therefore be regarded as being appropriate. To determine the discriminant validity of the model, we used the Fornell-Larcker criterion, the cross-loadings (Table 1), and additionally the heterotrait monotrait ratio of correlations (HTMT), which is used due to the criticism of the use of the Fornell-Larcker criterion for variant-based SEM [44]. The discriminant validity analysis ensures that the latent constructs are empirically independent and therefore only measure the empirical variables to which they are associated [40]. As indicated in Table 2, the analysis showed smaller correlations between the latent variables compared to the square root values of AVE [43].

Table 2. Measurement model statistics and AVEs

\begin{tabular}{|c|c|c|c|c|c|c|c|c|c|}
\hline Construct & C.R. & C.A. & AVE & 1 & 2 & 3 & 4 & 5 & 6 \\
\hline PEOU & 0.946 & 0.924 & 0.814 & \begin{tabular}{|c|}
0.902 \\
$\div * * *$
\end{tabular} & & & & & \\
\hline EXP & 1.000 & 1.000 & 1.000 & -0.011 & $\begin{array}{l}1.000 \\
* * * *\end{array}$ & & & & \\
\hline \begin{tabular}{|l|} 
INT_USE \\
\end{tabular} & 0.846 & 0.727 & 0.648 & 0.323 & -0.095 & $\begin{array}{l}0.805 \\
* * * *\end{array}$ & & & \\
\hline PU & 0.916 & 0.890 & 0.647 & 0.414 & -0.044 & 0.663 & $\mathbf{D}_{* * *}^{0.804}$ & & \\
\hline PRIV & 0.941 & 0.917 & 0.800 & $\mid-0.159$ & 0.030 & $\mid-0.279$ & -0.293 & $\begin{array}{l}0.895 \\
* * *\end{array}$ & \\
\hline TR & 0.898 & 0.857 & 0.639 & 0.400 & $-0,038$ & 0.489 & 0.481 & -0.454 & $\begin{array}{l}\mathbf{0 . 7 9 9} \\
* * *\end{array}$ \\
\hline
\end{tabular}

Likewise, the cross-loading examination revealed that the loads of all indicators are by far the strongest on their own construct (Table 1) [38]. In addition, the HTMT shows a consistent undercutting of the threshold value of 0.85 [40]. In our model, the values for all the constructs range from 0.031 to 0.809 . Both the HTMT and the Fornell-Larcker criterion show evidence of discriminant validity [44]. The latent constructs can therefore all be described as onedimensional, reliable and valid. Once the evaluation of the reliability and validity of the measurement model was fulfilled, before testing our hypothesis, the variance inflation factor (VIF) was used to test the structural model for multi-collinearity and common method bias (CMV) [45]. As indicated by Kock [46, p. 7] "[...] a VIF greater than 3.3 is 
proposed as an indication of pathological collinearity, and also as an indication that a model may be contaminated by common method bias". Since, the VIF values of all constructs in our model range between 1.003 and 1.617 , it can be concluded that the structural model does not show signs of common method bias or multi-collinearity problems. Hence, after having completed all the tests described above, we proceeded to test our hypotheses. Figure 1 depicts the PLS-SEM path coefficients and their significances (p-values), as well as the theoretically assumed relationships between the constructs [40], i.e. t-values, determined using a bootstrap procedure based on 3000 replications [44].

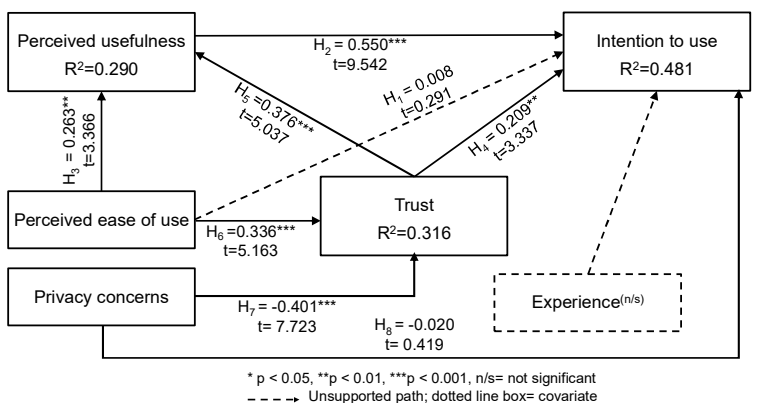

Figure 1. Partial least squares results for the structural model

To analyze the practical significance of the structural model, we assessed whether the independent latent variables exert a substantial influence on the dependent variables by analyzing the coefficients of determination $\left(\mathrm{R}^{2}\right)$ of the endogenous constructs [44]. The analysis of $\mathrm{R}^{2}$ indicates that $48.1 \%$ of the variance in the intention to use ICB can be jointly explained by the constructs of perceived usefulness, perceived ease of use, privacy concerns and trust. Furthermore, the model estimation shows that the construct of perceived usefulness can be $29.0 \%$, explained by the constructs of perceived ease of use and trust, whereas the perceived ease of use and privacy concerns constructs can explain $31.6 \%$ of the variance in the endogenous construct of trust. The PLS structural model results (Table 3) also show that perceived usefulness has a stronger positive effect than trust and perceived ease of use on the intention to use, while privacy concerns have a negative significant effect on trust, but not a significant negative effect on the intention to use ICB. As shown in Table 3, the results support six of our hypotheses. Regarding experience (covariate), we find no significant relationship with the intention to use. Furthermore, we checked for a moderating effect of experience between trust and the intention to use. Our analysis shows that experience does not moderate the relationships between trust and the intention to use ( $\mathrm{t}-$ statistic $(|\mathrm{O} / \mathrm{STDEV}|=1.845$; $\mathrm{p}$-value $=0.065)$.
The effect size $\mathrm{f}^{2}$ is a further indicator of the degree of influence of exogenous latent variables on the latent endogenous variables [38]. The effect size, according to Cohen [47], is calculated to check for practical significance. Guidelines for assessing $\mathrm{f}^{2}$ are the values $0.02-0.14$ for small effects, $0.15-0.34$ for medium effects, and above 0.35 for large effects [47]. The effect size of the construct perceived usefulness on the variable intention to use should be evaluated as prominent. The effect size for the relationship of trust to perceived usefulness is medium. This result also applies to the relationship between the perceived ease of use and privacy concerns on trust. The effect size between trust and the intention to use ICB as well as the effect size between perceived ease of use and perceived usefulness show a small effect (Table 3 ).

Table 3. Partial least squares results and measurement model statistics

\begin{tabular}{|l|l|c|c|c|c|c|}
\hline $\mathbf{H}_{\mathbf{n}}$ & Relationship & $\boldsymbol{\beta}$ & T-value & P-value & $\mathbf{f}^{2}$ & Results \\
\hline $\mathrm{H}_{1}$ & PEOU $\rightarrow$ INT_USE & 0.008 & 0.291 & 0.771 & 0.000 & Not supported \\
\hline $\mathrm{H}_{2}$ & PU $\rightarrow$ INT_USE & 0.550 & 9.542 & 0.000 & 0.409 & Supported \\
\hline $\mathrm{H}_{3}$ & PEOU $\rightarrow$ PU & 0.263 & 3.366 & 0.001 & 0.082 & Supported \\
\hline $\mathrm{H}_{4}$ & TR $\rightarrow$ INT_USE & 0.209 & 3.337 & 0.001 & 0.052 & Supported \\
\hline $\mathrm{H}_{5}$ & TR $\rightarrow$ PU & 0.376 & 5.037 & 0.000 & 0.167 & Supported \\
\hline $\mathrm{H}_{6}$ & PEOU $\rightarrow \mathrm{TR}$ & 0.336 & 5.163 & 0.000 & 0.161 & Supported \\
\hline $\mathrm{H}_{7}$ & PRIV $\rightarrow$ TR & -0.401 & 7.723 & 0.000 & 0.229 & Supported \\
\hline $\mathrm{H}_{8}$ & PRIV $\rightarrow$ INT_USE & -0.020 & 0.419 & 0.675 & 0.001 & Not supported \\
\hline
\end{tabular}
Perceived Usefulness; TR: Trust; PRIV: Privacy Concerns

Note: $\mathrm{H}=$ Hypothesis; $\beta=$ path coefficient; Cohen's $\mathrm{f}^{2}$-statistics $=\left[\mathrm{R}^{2}\right.$ incl. $-\mathrm{R}^{2}$ excl. $] /[1-$ $\mathrm{R}^{2}$ incl.] (1988); $\mathrm{f}^{2} \geq 0.02,0.15$ and 0.35 correspond to small, medium, and large effects.

\section{Discussion, implications, limitations and further research}

Our research examines the influencing factors on the intention to use ICB. Our results show that as predicted, trust has a positive significant influence on the users' desire to interact with a chatbot system to accomplish a specific goal or task related to insurance services $\left(\mathrm{H}_{4}\right)$. This is in line with the findings of Kasilingam et al. [21] in the context of shopping chatbots. However, the effect size for the relationship of trust to intention to use ICB in our analysis is small and is not restrictive to inexperience individuals. Conversely, our analysis indicates that the effect of perceived usefulness on the variable intention to use is prominent $\left(\mathrm{H}_{2}\right)$, while at the same time evidences a significant positive influence between trust and the perceived usefulness of ICB $\left(\mathrm{H}_{5}\right)$. The above findings suggest the existence of context-related preferences in the trade-off between practical use and trust. In the specific case of ICB, this implies that users are willing to rely upon $\mathrm{ICB}$, if they consider the expected 
result, e.g., to report an insurance claim, as high enough to make the effort to interact with the ICB [5].

Moreover, our examination has further found that the perceived ease of use has a significant positive influence both on the perceived usefulness $\left(\mathrm{H}_{3}\right)$ and on trust $\left(\mathrm{H}_{6}\right)$, but its influence is not significant on the intention to use of ICB $\left(\mathrm{H}_{1}\right)$. With regard to the latter, diverse meta-analyses of the TAM have found empirical evidence suggesting a usually weak and not significant influence of perceived ease of use on intention to use (e.g., [48, 49]) in studies where either similar measurement items have been used to assess perceived usefulness and perceived ease of use, or the path coefficients have been measured using sample sizes with less than 225 observations. Since our sample size consists of 215 respondents, the effect of perceived ease of use on intention to use can be partially mediated by or attributed to perceived usefulness (e.g., [48]). For our analysis, this indicates that the perceived ease of use is an enabling factor of the cognitive trust building process [15]. However, even when the design of a ICB is simple, clear, understandable and easy to learn, if the operational outcomes and expected benefits do not match the user's cognitive trust expectations, the ICB will not contribute to optimize the customer experience, due to the customer's difficulty in discerning the added value of an interaction through a computerized text-based dialog system instead of a human agent [9].

On the other hand, as conjectured, privacy concerns have a significant negative influence on trust in ICB $\left(\mathrm{H}_{7}\right)$. Consequently, privacy concerns about ICB generate in the users a perception of intransparency and loss of control within the humanchatbot interactive process and, therefore act as inhibitors of trust-building [17]. Nevertheless, although the negative influence of privacy concerns on the behavioral intention to use has been found to be significant in studies related to the context of shopping chatbots (see e.g., [22]), against the expectations, the effect of privacy concerns on the intention to use ICB $\left(\mathrm{H}_{8}\right)$ could not be supported. The explanation for this may lie in a psychological effect on the assessment of data protection risks, as a result of the implementation of the European General Data Protection Regulation (GDPR) in 2018, which imposes high economic penalties of up to 20 million euros or $4 \%$ of the global annual revenue on non-compliant companies [50].

Based on the findings of our analysis, the following potential design implications can be drawn: (i) functional and effort efficient chatbot design: In other applications domains the presence of anthropomorphic features in chatbots has been suggested to be equally or even more critical for their acceptance, than functional features (e.g., [8, 18]).
However, the presence of functional features enhancing the user experience and the perception of usefulness are of upmost importance in ICB acceptance. Hence, ICB design must tend to goaloriented interactions in which the ICB takes the role of facilitator offering graphical response elements to reduce the interaction effort, and the integration of multiple services to improve content customization; (ii) higher visibility of privacy and data protection efforts: Our findings suggest that privacy concerns have a negative impact on trust in ICB. Although our empirical evidence indicates that trust is not the critical factor behind the intention to use ICB, it is an important weighting mechanism for framing the rational judgment upon the functional characteristics or qualities of ICB. However, our explorative analysis of real-world German ICB (see Section 2.1) showed that many of analyzed ICB do not display or request a confirmation of the privacy policy at the beginning of the interaction. Yet, to positively shape the users' privacy and protection beliefs, an ICB not only must achieve actual privacy and data protection, but also the appearance of it. Therefore, we recommend insurance companies to give higher level of visibility to their undertaken privacy and data protection efforts and offer users the possibility to contact a human agent through the ICB in case of possible concerns about unauthorized secondary use and information control; (iii) user-centered rather than design-push chatbots: Standard design approaches are mostly centered on the needs and vision of the implementing companies. However, design efforts should be focused on strengthen the users' perceived usefulness. This can be accomplished by enhancing the co-innovation of insurance products and services through the application of user-centered chatbot implementation strategies in which the future user is taken into account throughout the chatbot development to ensure that practical valueadded chatbot design elements are prioritized.

The limitations of our work could include the exclusive investigation of the German-speaking area. Potential cultural differences cannot be identified in this way. Whereas the findings of previous trust research do not currently indicate that major cultural differences are to be expected (e.g. [5]), in terms of privacy concerns might be country-specific differences. An explicit investigation of cultural differences, especially in the context of ICB attempting to provide more human-like interactions through advanced NLP techniques, could make a key scientific contribution.

Further research can use the developed SEM to investigate additional possible moderators that could influence the intention to interact with ICB (e.g., age, gender, type of insurance service used). As well, since 
the majority of the survey respondents in our study have not direct experience with ICB, but possess former cognitive trust expectations in relation to the use of chatbots in other domains. A following study can combine our study with an online experiment in which, for example, participants test the ICB classified in section 2.1 before conducting the survey. In this context, it would also be advisable to adapt the constructs to other business areas and to carry out a cross-domain analysis to identify differences between the application areas.

We also recommend investigating the extent to which trust influences intention to use, especially in other industries where a high level of confidentiality is appropriate and sensitive data is processed, e.g., healthcare or asset management. Such cross-industry analysis could contribute to generate interdisciplinary practical knowledge which can be further integrated into user-oriented chatbot implementation frameworks.

\section{Conclusion}

Our goal was to investigate how perceived ease of use, perceived usefulness, trust, and privacy concerns directly or indirectly influence the intention to use German ICB. Through conducting an online survey with 215 respondents and PLS-SEM analysis, we found that, despite trust has a significant positive influence on the intention to use, the perceived usefulness has a greater positive influence on the intention to interact with ICB. This implies that features that bring practical added value to the digital customer experience are most critical for ICB usage.

\section{References}

[1] M. Jain, P. Kumar, R. Kota, and S. N. Patel. "Evaluating and Informing the Design of Chatbots", In Proc. Designing Interactive Systems Conference, 2018, pp. 895-906.

[2] A. Janssen, J. Passlick, D. Rodríguez Cardona, and M. H. Breitner. "Virtual Assistance in any Context: A Taxonomy of Design Elements for Domain-specific Chatbots", Business \& Information Systems Engineering, 62(3), 2020, pp. 211-225.

[3] Helpshif. State of Customer Service Automation 2019, 2019. https://go.helpshift.com/rs/113-UDX-599/ images /Report_State_of_CS_Automation.pdf, Accessed: 15 June 2020.

[4] I. Sebastian, J. Ross, C. Beath, M. Mocker, K. Moloney, and N. Fonstad. "How Big Old Companies Navigate Digital Transformation”, MIS Quarterly, 16(3), 2017, pp. 197-213.

[5] S. Gebert-Persson, M. Gidhagen, J. E. Sallis, and H. Lundberg. "Online Insurance Claims: When More than Trust Matters", International Journal of Bank Marketing, 37(2), 2019, pp. 579-594.
[6] D. Rodríguez Cardona, O. Werth, S. Schönborn, and M. H. Breitner. "A Mixed Methods Analysis of the Adoption and Diffusion of Chatbot Technology in the German Insurance Sector", In Proc. American Conference on Information Systems, 2019.

[7] A. Przegalinska, L. Ciechanowski, A. Stroz, P. Gloor, and G. Mazurek. "In Bot We Trust: A New Methodology of Chatbot Performance Measures", Business Horizons 62(6), 2019, pp. 785-797.

[8] L. Müller, J. Mattke, C. Maier, T. Weitzel, and H. Graser. "Chatbot Acceptance: A Latent Profile Analysis on Individuals' Trust in Conversational Agents", In Proc. Computers and People Research Conference, 2019, pp. $35-42$.

[9] N. V. Wuenderlich, and S. Paluch. "A Nice and Friendly Chat with a Bot: User Perceptions of AI-based Service Agents", In Proc. International Conference on Information Systems, 2017.

[10] A. Singh, K. Ramasubramanian, and S. Shivam. "Processes in the Banking and Insurance Industries", In: Building an Enterprise Chatbot. Apress, Berkeley, CA, 2019.

[11] F. Koetter, M. Blohm, J. Drawehn, M. Kochanowski, J. Goetzer, D. Graziotin, and S. Wagner. "Conversational Agents for Insurance Companies: From Theory to Practice", In Proc. International Conference on Agents and Artificial Intelligence, Lecture Notes in Computer Science, 11978. Springer, Cham, 2019, pp. 338-362.

[12] M. Riikkinen, H. Saarijärvi, P. Sarlin, and I. Lähteenmäki. "Using Artificial Intelligence to Create Value in Insurance", International Journal of Bank Marketing, 36(6), 2018, pp. 1145-1168.

[13] H. J. Watson and C. Nations. "Addressing the Growing Need for Algorithmic Transparency", Communications of the Association for Information Systems, 45(1), 2019, pp. 488-510.

[14] D. M. Rousseau, S. B. Sitkin, R. S. Burt, and C. Camerer. "Not so Different after All: A Cross-Discipline View of Trust", Academy of Management Review, 23(3), 1998, pp. 393-404.

[15] H. Fan, R. Lederman, S. P. Smith, and S. Chang. "How Trust is Formed in Online Health Communities: A Process Perspective", Communications of the Association for Information Systems, 34(1), 2014, pp. 531-560.

[16] J. Y. Jian, A. M. Bisantz, and C. G. Drury. "Foundations for an Empirically Determined Scale of Trust in Automated Systems", International Journal of Cognitive Ergonomics, 4(1), 2000, pp. 53-71.

[17] D. H. Mcknight, M. Carter, J. B. Thatcher, and P. F. Clay. "Trust in a Specific Technology: An Investigation of its Components and Measures", Transactions on Management Information Systems, 2(2), 2011, pp. 1-13.

[18] D. R. Large, K. Harrington, G. Burnett, J. Luton, P. Thomas, and P. Bennett. "To Please in a Pod: Employing an Anthropomorphic Agent-Interlocutor to Enhance Trust and User Experience in an Autonomous, SelfDriving Vehicle", In Proc. 11th International Conference on Automotive User Interfaces and Interactive Vehicular Applications, 2019, pp. 49-59.

[19] A. Følstad, C. B. Nordheim, and C. A. Bjørkli. "What Makes Users Trust a Chatbot for Customer Service? An 
Exploratory Interview Study”, In Proc. International Conference on Internet Science, Lecture Notes in Computer Science, 11193. Springer, Cham, 2018, pp. 194-208.

[20] S. Laumer, C. Maier, and F. T. Gubler. "Chatbot Acceptance in Healthcare: Explaining User Adoption of Conversational Agents for Disease Diagnosis", In Proc. European Conference on Information Systems, 2019.

[21] D. L. Kasilingam. "Understanding the Attitude and Intention to Use Smartphone Chatbots for Shopping", Technology in Society, 62, 2020, pp.1-15.

[22] A. Rese, L. Ganster, and D. Baier. "Chatbots in Retailers' Customer Communication: How to Measure their Acceptance?" Journal of Retailing and Consumer Services, 56, 2020, pp. 1-14.

[23] F. D. Davis. "Perceived Usefulness, Perceived Ease of Use, and User Acceptance of Information Technology", MIS Quarterly, 13(3), 1989, pp. 319-340.

[24] V. Venkatesh and F. D. Davis. "A Theoretical Extension of the Technology Acceptance Model: Four Longitudinal Field Studies", Management Science, 46(2), 2000, pp. 186-204.

[25] V. Venkatesh, M. G. Morris, G. B. Davis and F. D. Davis. "User Acceptance of Information Technology: Toward a Unified View", MIS Quarterly, 27(3), 2003, pp. 425-478.

[26] P. A. Pavlou. "Consumer Acceptance of Electronic Commerce: Integrating Trust and Risk with the Technology Acceptance Model", International Journal of Electronic Commerce, 7(3), 2003, pp. 101-134.

[27] N. Guhr, O. Werth, P. P. H. Blacha and M. H. Breitner. "Privacy Concerns in the Smart Home Context", SN Applied Sciences, 2(247), 2020, pp. 1-12.

[28] N. M. Baba, A. S. Baharudin and S. Alomari. "Determinants of Users' Intention to use Smartwatch", Journal of Theoretical and Applied Information Technology, 97(18), 2019, pp. 4738-4750.

[29] M. Fishbein, and I. Ajzen. "Predicting and Understanding Consumer Behavior: Attitude-Behavior Correspondence", Understanding Attitudes and Predicting Social Behavior, 1980, pp. 148-172.

[30] A. Verbovetska. "The Impact of Financial Technology on Customer Intention to Use Financial Services through the Lenses of Process Virtualization Theory", In Proc. International Conference on Information Systems, 2019.

[31] P. Pu, L. Chen, and R. Hu. "Evaluating Recommender Systems from the User's Perspective: Survey of the State of the Art", User Modeling and User-Adapted Interaction, 22(4-5), 2012, pp. 317-355.

[32] D. Gefen, and D. Straub. "Managing User Trust in B2C E-Services", e-Service 2(2), 2003, pp. 7-24.

[33] N. K. Lankton, D. H. McKnight, and J. Tripp. "Technology, Humanness, and Trust: Rethinking Trust in Technology", Journal of the Association for Information Systems, 16(10), 2015, pp. 880-918.

[34] C. Stephanidis, G. Salvendy, M. Antona, Y. J Chen, J. Dong, V. G. Duffy, and Y. Guo. "Seven HCI Grand Challenges", International Journal of Human-Computer Interaction, 35(14), 2019, pp. 1229-1269.
[35] A. Ho, J. Hancock, and A. S. Miner. "Psychological, Relational, and Emotional Effects of Self-disclosure after Conversations with a Chatbot", Journal of Communication, 68(4), 2018, pp. 712-733.

[36] T. Dinev, H. Xu, J. H. Smith, and P. Hart. "Information Privacy and Correlates: An Empirical Attempt to Bridge and Distinguish Privacy-related Concepts", European Journal of Information Systems, 22(3), 2013, pp. 295316.

[37] M. S. Featherman, A. D. Miyazaki, and D. E. Sprott. "Reducing Online Privacy Risk to Facilitate E-service Adoption: The Influence of Perceived Ease of Use and Corporate Credibility", Journal of Services Marketing, 24(3), 2010, pp. 219-229.

[38] W. W. Chin. "The Partial Least Squares Approach to Structural Equation Modeling", Modern Methods for Business Research, 295(2), 1998, pp. 295-336.

[39] C. Nitzl. "The Use of Partial Least Squares Structural Equation Modelling (PLS-SEM) in Management Accounting Research: Directions for Future Theory Development", Journal of Accounting Literature, 37, 2016, pp. 19-35.

[40] J. F. Hair Jr., G. T. M. Hult, C. Ringle, and M. Sarstedt. A Primer on Partial Least Squares Structural Equation Modeling (PLS-SEM), Sage publications, 2016.

[41] E. Cho. "Making Reliability Reliable: A Systematic Approach to Reliability Coefficients", Organizational Research Methods, 19(4), 2016, pp. 651-682.

[42] A. Diamantopoulos, P. Riefler, and K. P. Roth "Advancing Formative Measurement Models", Journal of Business Research, 61(12), 2008, pp. 1203-1218.

[43] C. Fornell, and D. F. Larcker. "Evaluating Structural Equation Models with Unobservable Variables and Measurement Error", Journal of Marketing Research, 18(1), 1981, pp. 39-50.

[44] J. Henseler, C. M. Ringle, and M. Sarstedt. "A New Criterion for Assessing Discriminant Validity in Variance-based Structural Equation Modeling", Journal of the Academy of Marketing Science, 43(1), 2015, pp. 115-135.

[45] S. J. Chang, A. Van Witteloostuijn, and L. Eden. "From the Editors: Common Method Variance in International Business Research", Journal of International Business Studies, 41(2), 2010, pp. 178-184.

[46] N. Kock. "Common Method Bias in PLS-SEM: A Full Collinearity Assessment Approach". International Journal of e-Collaboration, 11(4), 2015, pp. 1-10.

[47] Cohen, J. Statistical Power Analysis for the Behavioral Sciences, Hillsdale, New Jersey: Lawrence Erlbaum Associates, Publishers, 1988.

[48] W. R. King, and J. He. "A Meta-analysis of the Technology Acceptance Model", Information \& Management, 43(6), 2006, pp. 740-755.

[49] C. Feng, X. Su, Z. Lin, Y. He, N. Luo, and Y. Zhang. "Determinants of Technology Acceptance: Two Modelbased Meta-analytic Reviews", Journalism \& Mass Communication Quarterly, 2020, pp. 1-22.

[50] European Commission. Data protection in the EU, 2016. https://ec.europa.eu/info/law/law-topic/data-protection/ dataprotection-eu en. Accessed: 27 September 2020. 